\title{
Bilan de 15 ans de radiocancérologie expérimentale au CEA
}

\author{
J. LAFUMA * \\ (Manuscrit reçu le 7 décembre 1982)
}

II y a quinze ans, un programme de recherche expérimentale sur l'action cancérigène des rayonnements ionisants et notamment des particules à TEL élevé ( $\alpha$, neutrons) fut entrepris au Département de protection.

L'objectif était, d'abord, de comparer la sensibilité d'une souche de rat avec celle de l'homme dans des conditions d'irradiation aussi semblables que possible dans les deux espèces. Ce but étant atteint, cette souche de rat devait être soumise à des modes d'irradiation pour lesquelles il n'existait aucune donnée humaine. Les données obtenues permettraient, par comparaison avec le cas choisi comme référence, de fixer le risque humain. Comme référence, on choisit, à l'époque, les inhalations de radon 222 , ceci pour trois raisons principales :

- les seules données humaines en irradiations professionnelles susceptibles d'être acquises dans les deux ou trois décades à venir, proviendraient vraisemblablement des mines d'uranium ;

- il existait, en France, des mines d'uranium, ce qui justifiait un intérêt tout spécial dans ce domaine, mais aussi permettait de monter en Limousin un laboratoire équipé pour ce type d'expériences ;

- enfin, la France se lançait dans des programmes militaires et civils basés sur l'emploi du plutonium, pour lequel le risque principal est également l'induction de cancers pulmonaires. II semblait logique de prendre comme référence un autre émetteur $\alpha$ inhalé plutôt que des irradiations externes avec des rayons $X$.

Au cours des quinze dernières années, ce programme s'est développé :

- les études sur le radon se terminent et la dose la plus faible utilisée $(200 \mathrm{WLM})^{\star \star}$ correspond à 5 ans de travail à la dose maximale admissible humaine ;

- les études sur les actinides s'orientent maintenant vers des recherches sur les mécanismes de l'action cancérigène et vers l'étude de formes très spéciales de plutonium ;

* Commissariat à l'énergie atomique, IPSN, Département de protection, BP 6 , 92260 Fontenay-aux-Roses.

** WLM (working level month) : 1 WLM équivaut à 3,6 millijoules par mètre cube par heure. 
- les expériences avec les neutrons de fission sont terminées. La dose absorbée la plus faible $(0,016 \mathrm{~Gy})$ correspond à trois années de travail à la dose maximale admissible humaine ;

- quelques expériences avec les $\gamma$ ont été lancées, mais elles montrent qu'avec nos techniques expérimentales, il ne sera pas possible de descendre au-dessous de 1 Gy.

Ce programme, qui se déroule depuis quinze ans, est donc pratiquement achevé. II est possible de commencer à en faire le bilan qui, en toute logique, doit débuter par une synthèse des résultats acquis avec l'élément de référence : le radon 222.

Ensuite, seront exposées les expériences menées avec les irradiations globales neutroniques. Les relations dose-effets seront analysées pour les différents cancers. Dans chaque cas, on comparera l'action sur l'homme et l'action sur le rat.

Un troisième article sera consacré à l'action cancérigène des actinides, qu'ils aient été inhalés ou injectés. On comparera, pour un même organe, l'efficacité des particules $\gamma$ et celle des neutrons. Le rôle de l'hétérogénéité de la distribution de la dose dans l'organe sera analysé.

Enfin, dans un quatrième article, on exposera les recherches pratiques pour comprendre, à l'échelle de l'organisme ou de l'organe, les mécanismes de l'induction des cancers par les rayonnements. 